\title{
Noise Removal in Microarray Images Using Variational Mode Decomposition Technique
}

\author{
G. Sai Chaitanya Kumar*1, Reddi Kiran Kumar², G. Apparao Naidu ${ }^{3}$, J. Harikiran ${ }^{4}$ \\ ${ }^{1}$ Dept of CSE, JNTU Hyderabad, India \\ ${ }^{2}$ Dept of CS, Krishna University, Machilipatnam, India \\ ${ }^{3}$ Dept of CSE, J.B. Institute of Engineering and Technology, Hyderabad, India \\ ${ }^{4}$ Department of IT, GIT, GITAM University, Visakhapatnam, India \\ *Corresponding author, e-mail: saijntuhphd@gmail.com
}

\begin{abstract}
Microarray technology allows the simultaneous monitoring of thousands of genes in parallel. Based on the gene expression measurements, microarray technology have proven powerful in gene expression profiling for discovering new types of diseases and for predicting the type of a disease. Enhancement, Gridding, Segmentation and Intensity extraction are important steps in microarray image analysis. This paper presents a noise removal method in microarray images based on Variational Mode Decomposition (VMD). VMD is a signal processing method which decomposes any input signal into discrete number of sub-signals (called Variational Mode Functions) with each mode chosen to be its band width in spectral domain. First the noisy image is processed using 2-D VMD to produce 2-D VMFs. Then Discrete Wavelet Transform (DWT) thresholding technique is applied to each VMF for denoising. The denoised microarray image is reconstructed by the summation of VMFs. This method is named as 2-D VMD and DWT thresholding method. The proposed method is compared with DWT thresholding and $B E M D$ and DWT thresholding methods. The qualitative and quantitative analysis shows that 2-D VMD and DWT thresholding method produces better noise removal than other two methods.
\end{abstract}

Keywords: Empirical Mode decomposition, Variational Mode Decomposition, Discrete Wavelet Transform, Image Enhancement, Microarray Images

Copyright $(2017$ Universitas Ahmad Dahlan. All rights reserved.

\section{Introduction}

The most powerful tool in molecular genetics for biomedical research is Microarray, which allows parallel analysis of the expression level of thousands of genes. The most important aspect in microarray experiment is image analysis. The output of image analysis is a matrix consisting of a measure of intensity of each spot in the image. This measure denotes gene expression ratio (transcription abundance) between the test and control samples for the corresponding gene. The positive expression indicates the over-expression, while negative expression indicates under-expression between the control and treatment genes. The main components in microarray image analysis are localization, segmentation and spot quantification [1]. This paper mainly focuses on Gaussian noise removal in microarray images. The main applications of microarray technology are Gene discovery, Drug discovery, Disease diagnosis, Toxicological research etc [2]. The microarray image analysis is shown in Figure 1.

The evaluation of microarray images is a difficult task as the fluorescence of the glass slide adds noise floor to the microarray image. The processing of the microarray image requires noise suppression with minimal reduction of spot edge information that derives the segmentation process. Thus the task of microarray image enhancement is of paramount importance [3]. This paper presents a Gaussian noise removal in microarray images using Variational Mode Decomposition. First the noisy image is decomposed in to VMFs using 2-D VMD. Each VMF is denoised using DWT thresholding technique. After denoising, the image is reconstructed by combining all 2-D VMFs. After noise removal, gridding, segmentation and Expression ratio calculation are the important tasks in microarray image analysis [4]. Any noise in the microarray image will affect the subsequent analysis. The paper is organized as follows: Section 2 presents Denoising using DWT Thresholding method, Section 3 presents Denoising using Bi-dimensional Empirical Mode Decomposition (BEMD) and DWT thresholding method, 
Section presents Denoising using 2-D VMD and DWT thresholding method, Section 5 presents FCM Clustering Algorithm, Section 6 presents Experimental results and Section 7 report conclusions.

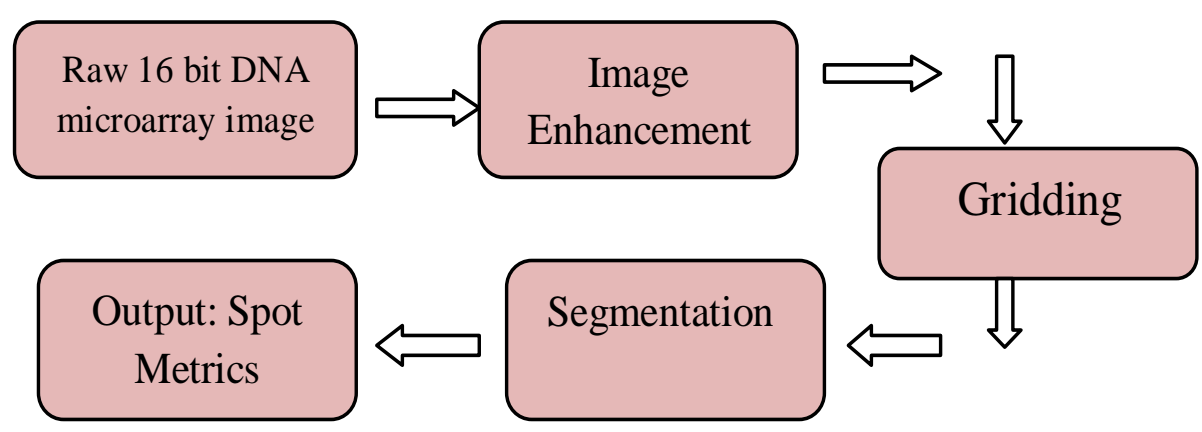

Figure 1. Microarray Image Analysis

\section{Discrete Wavelet Transform (DWT) Thresholding Method}

The algorithm of microarray image denoising using Discrete Wavelet Transform (DWT) is summarized as follows:

a. Perform multiscale decomposition [5] of the image corrupted by Gaussian noise using wavelet transform.

b. Estimate the noise variance $\sigma^{2}$. The noise variance is estimated from sub-band $\mathrm{HH} 1$ using the formula

$$
\sigma^{2}=\left[\frac{\text { median }\left(\left|y_{i j}\right|\right.}{0.6745}\right]^{2}, y_{i j} \in \text { subbandHH1 }
$$

c. For each level, compute the scale parameter $\beta$ using [6]

$$
\beta=\sqrt{\log \frac{L_{k}}{J}} L_{k} \text { is the length of the sub-band at } k^{\text {th }} \text { scale. }
$$

d. For each sub-band (except the low pass residual)

1. Compute the standard deviation $\sigma_{y}$.

2. Apply soft thresholding to the noisy coefficients. The thresholds used by wavelets for filtering are universal threshold [7], SURE shrink [8], Bayes shrink [9] and Normal shrink [10].

e. Invert the multiscale decomposition to reconstruct the denoised image.

\section{BI-Dimensional Empirical Mode Decomposition and DWT Thresholding Method}

Empirical mode decomposition [11] is a signal processing method which divides any signal into number of oscillatory functions called intrinsic mode functions (IMF). Each IMF satisfies two properties given in [12]. The first IMF is high frequency component and last IMF is low frequency component.

The shifting process [13] used to obtain IMFs on a 2-D signal (microarray image) is summarized as follows:

a. Find all local maxima and local minima points in $I(x, y)$.

b. Interpolate the local maxima and local minima points using cubic spline interpolation method to create envelopes. $(U p(x, y)$ upper envelope and $L w(x, y)$ lower envelope) 
$\operatorname{Mean}(x, y)=\frac{(U p(x, y)+\operatorname{Lw}(x, y))}{2}$

c. $\quad \operatorname{Sub}(x, y)=I(x, y)-\operatorname{Mean}(x, y)$

d. $\quad \operatorname{IMF}_{i}(x, y)=\operatorname{Sub}(x, y)$

e. $\quad I(x, y)=I(x, y)-I M F_{i}(x, y)$

Repeat the above steps (b) to (f) for the generation of next IMFs.

$f$. This process is repeated until $\mathrm{I}(\mathrm{x}, \mathrm{y})$ does not have maxima or minima points to create envelopes.

Original Image can be reconstructed by inverse EMD given by

$$
I(x, y)=\sum_{i=1}^{n} I M F_{i}(x, y)+r e s(x, y)
$$

The mechanism of de-noising using BEMD and DWT is summarized as follows:

a. Apply 2-D EMD for noisy microarray to obtain IMFi $(i=1,2, \ldots k)$. The kth IMF is called residue.

b. The first few IMFs contains high frequency components and it is suitable for denoising and denoised with DWT Thresholding technique presented in section 2. This de-noised IMF is represented with DNIMF.

c. The denoised image is reconstructed by the summation of DNIMF and remaining IMFs given by

$$
R I=D N I M F 1+\sum_{i=2}^{k} I M F_{i}
$$

Where $\mathrm{RI}$ is the reconstructed band. The flow diagram of BEMD and DWT filtering is shown in Figure 2.

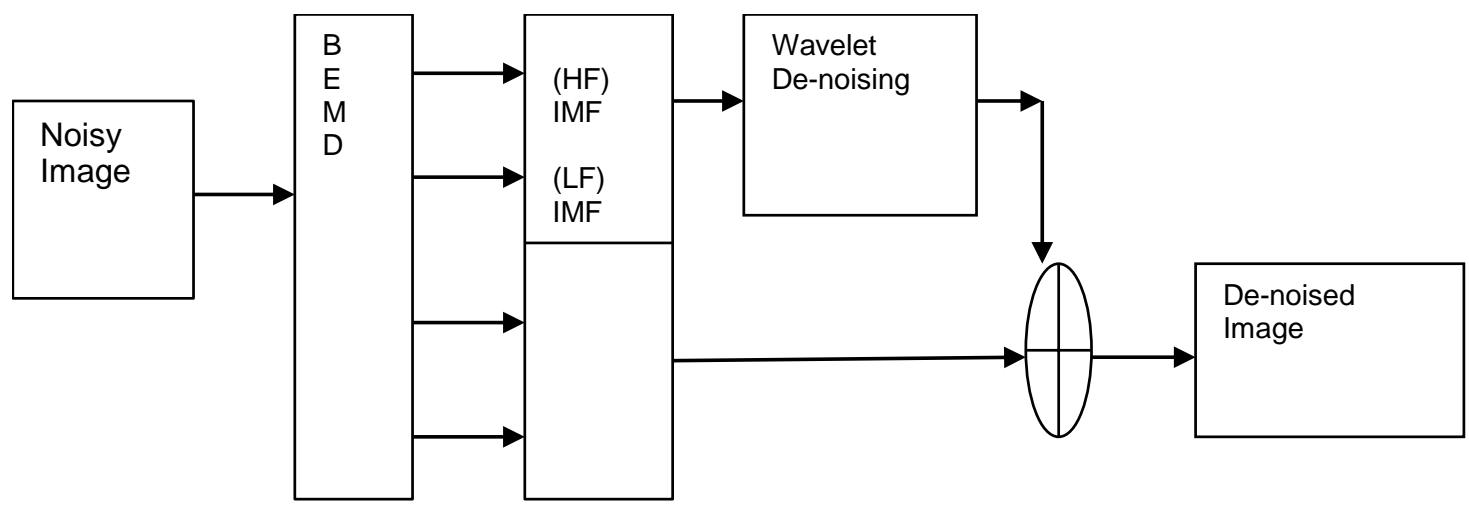

Figure 2. BEMD and DWT filtering

\section{Variational Mode Decomposition and DWT Threshonding Method}

The mechanism of de-noising using 2-D VMD and DWT is summarized as follows 
a. Apply 2-D VMD for noisy microarray to obtain VMFi ( $i=1,2, \ldots \mathrm{k})$. The $\mathrm{kth}$ VMF is called residue. The VMD framework is used to decompose any signal into k mode components presented in [15]

b. The VMFs are denoised with DWT Thresholding technique presented in section 2. This denoised VMF is represented with DNVMF.

c. The denoised image is reconstructed by the summation of VMFs given by

$$
R I=\sum_{i=1}^{k} V M F_{i}
$$

Where $\mathrm{RI}$ is the reconstructed image. The flow diagram of 2-D VMD and DWT filtering is shown in Figure 3.

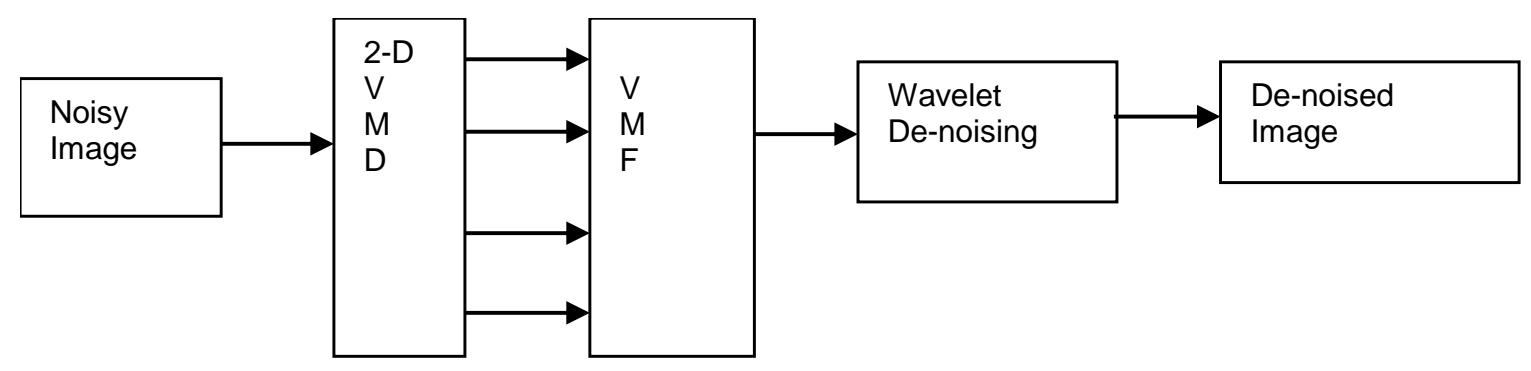

Figure 3. 2-D VMD and DWT filtering

\section{Fuzzy C-Means Clustering Algorithm}

1. Take $\mathrm{K}$ cluster centroid values [17]

2. Initialize membership matrix $u_{i j}$ and $m=2$.

3. Assign each pixel to the cluster according to the given formula [19].

$$
\begin{aligned}
& u_{i j}^{m} D\left(I_{i}, C_{j}\right)<u_{i q}^{m} D\left(I_{i}, C_{q}\right), q=1,2, \ldots, K \\
& j \neq q
\end{aligned}
$$

The new membership and cluster centroid values as calculated as [18]

$$
\begin{aligned}
u_{\hat{\mathrm{k}}} & =\frac{1}{\sum_{j=1}^{K}\left(\frac{D\left(C_{i}, I_{\dot{k}}\right)}{D\left(C_{j}, I_{\dot{k}}\right)}\right)^{\frac{1}{m-1}}}, \text { for } 1 \leq i \leq K \\
C_{j} & =\frac{\sum_{j=1}^{n} u_{\dot{j}}^{m} I_{j}}{\sum_{j=1}^{n} u_{i j}^{m}}
\end{aligned}
$$

4. Continue 2-3 until each pixel is assigned to the maximum membership cluster.

\section{Experimental Results}

In this section, the proposed filtering method is performed on a sample microarray slide drawn from the standard microarray database corresponds to breast category aCGH tumor tissue. To check the performance of the filtering methods presented in this paper Gaussian 
noise with different values of $\sigma$ is added to the image. The noisy image is filtered using the three methods presented in this paper. The quantitative results are evaluated using PSNR values are shown in Table 1 and qualitative results are shown in Figure 4. Both qualitative and quantitative results show that 2-D VMD and DWT filtering method has better denoising effect. After denoising the image is segmented using FCM clustering algorithm.

Table 1. PSNR Values of Filtering Method

\begin{tabular}{llll}
\hline Method & $\sigma=0.015$ & $\sigma=0.025$ & $\sigma=0.036$ \\
\hline Wavelet (Universal Threshold) & 22.02 & 16.98 & 14.89 \\
Wavelet (SURE shrink) & 21.90 & 16.11 & 13.76 \\
Wavelet (Bayes Shrink) & 20.08 & 15.72 & 12.91 \\
Wavelet (Normal Shrink) & 20.98 & 15.23 & 13.12 \\
BEMD + Wavelet (Universal Threshold) & 34.11 & 29.86 & 23.86 \\
BEMD + Wavelet (SURE shrink) & 32.89 & 28.74 & 22.11 \\
2-D VMD+ Wavelet (Bayes Shrink) & 36.16 & 30.98 & 24.43 \\
2-D VMD+ Wavelet (Normal Shrink) & 38.91 & 32.34 & 26.98 \\
\hline
\end{tabular}

Original Image (4a)

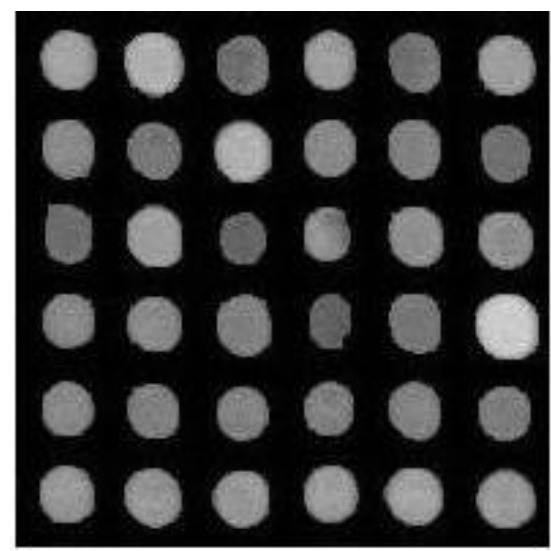

Filtering Using BEMD+DWT (4c)

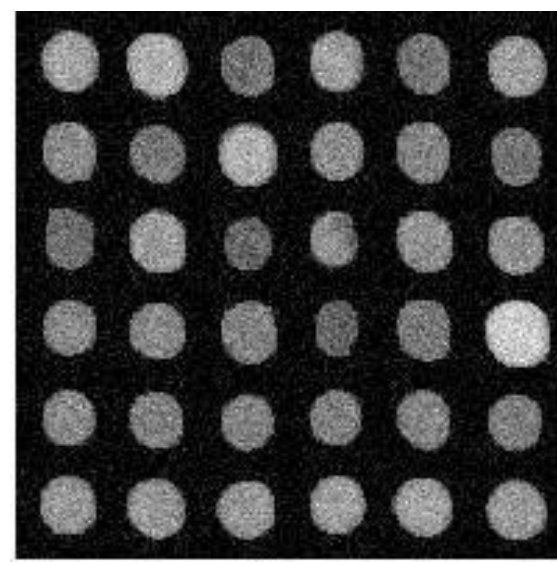

Segmentation using FCM on filtered image (4e)
Gaussian Noise (4b)

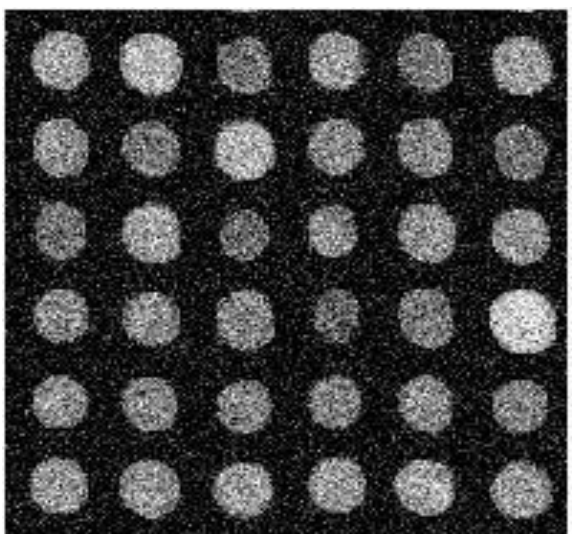

Filtering Using 2-D VMD+DWT (4d)

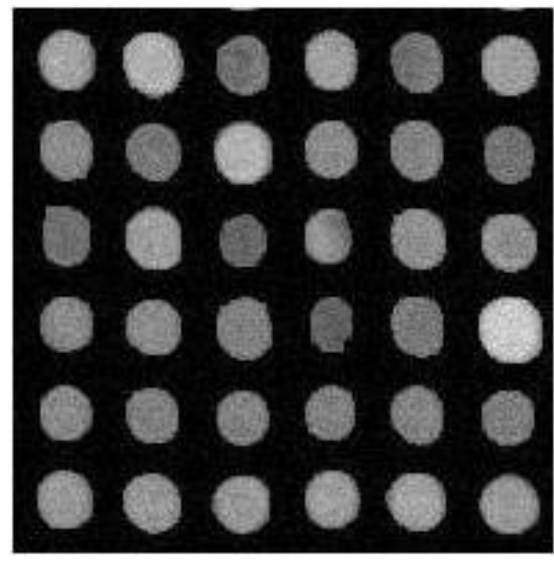

Segmentation using FCM on noisy image (4f) 

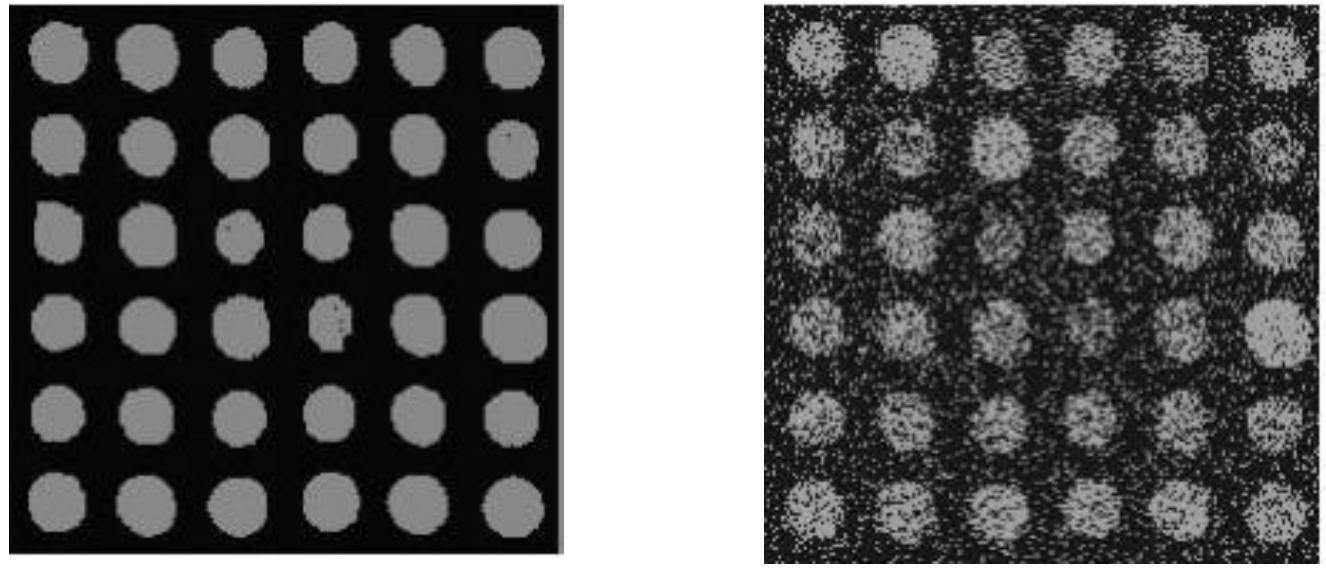

Figure 4. Qualitative Analysis of Filtering methods

\section{Conclusions}

Microarray technology is used for parallel analysis of gene expression ratio of different genes in a single experiment. The analysis of microarray image is done with gridding, segmentation and information extraction. The expression ratio of each and every gene spot denotes the transcription abundance between two genes under experiment. This paper presents different methods for microarray image denoising. Out of these methods 2-D VMD and DWT perform noise suppression in the image and produces better segmentation accuracy. Spot information includes the calculation of Expression Ratio in the region of every gene spot on the microarray image. The expression-ratio measures the transcription abundance between the two sample genes.

\section{References}

[1] J Harikiran, PV Lakshmi, R Kirankumar. Automatic Gridding Method for microarray images. Journal of Theoretical and Applied Information Technology. 2014; 6591: 235-241.

[2] J Harikiran, PV Lakshmi, R Kiran Kumar. Fast Clustering Algorithms for Segmentation of Microarray Images. International Journal of Scientific \& Engineering Research. 2014; 5(10): 569-574.

[3] J Harikiran, et.al. Vector Filtering Techniques for Impulse Noise Reduction with Application to Microarray images. International Journal of Applied Engineering Research. 2015; 10(3): 7181-7193.

[4] J Harikiran, et.al. A New Method of Gridding for Spot Detection in Microarray Images. Computer Engineering and Intelligent Systems. 2014; 5(3): 25-33.

[5] Savita Gupta and Lakhwinder Kaur. Wavelet Based Image Compression using Daubechies Filters. In proc. 8th National conference on communications, I.I.T. Bombay, NCC. 2002.

[6] Lakhwinder Kaur, Savita Gupta, RC Chauhan. Image Denoising using Wavelet Thresholding. Proceedings of ICVGIP. 2002.

[7] P Zhang, MD Desai. Adaptive denoising based on SURE risk. IEEE Signal Process. Lett. 1998; 5(10): 265-267.

[8] DL Donoho, IM Johnstone. Denoising by soft thresholding. IEEE Trans. on Iriform. Theory. 1995; 41: 613-627.

[9] TD Bui, GY Chen. Translation-invariant denoising using multiwavelets. IEEE Transactions on Signal Processing, 1998; 46(I2): 3414-3420.

[10] Sachin Ruikar, DD Doye. Image Denoising Using Wavelet Transform. 2010 International Conference on Mechanical and Electrical technology, ICMET. 2010.

[11] J Harikiran, et.al. Spot Edge detection in Microarray Images using Bi-dimensional Empirical Mode Decomposition. C3IT-2012, Elsevier Procedia Technology, (Science Direct). 2012; 4: 19-25.

[12] J Harikiran, PV Lakshmi, R Kiran Kumar. Multiple Feature Fuzzy C-means clustering algorithm for segmentation of microarray image. International Journal of Electrical and Computer Engineering (IJECE). 2015; 5(5): 1045-1053.

[13] B Saichandana, et.al. Image Fusion in Hyperspectral Image Classification using Genetic Algorithm. Indonesian Journal of Electrical Engineering and Computer Science (IJEECS). 2016; 2(3): 703-711.

[14] B Saichandana, J Harikiran, K Srinivas, R Kiran Kumar. Application of BEMD and Hierarchical Image Fusion in Hyper spectral Image Classification. International Journal of Computer Science and Information Security. 2016; 14(5): 437-445. 
[15] Salim Lahmiri, Mounir Boukadoum. Physiological Signal Denoising with Variational Mode decomposition and Weighted Reconstruction after DWT Thresholding. IEEE. 2015: 806-810.

[16] Konstantin Dragomiretskiy, Dominique Zosso. Variational Mode Decomposition. IEEE Transactions on Signal Processing. 2014; 62(3): 531-544.

[17] B Saichandana, et.al. Clustering Algorithm combined with Hill climbing for Classification of Remote Sensing Image. International Journal of Electrical and Computer Engineering (IJECE). 2014; 4(6): 923-930.

[18] J Harikiran et.al. Fuzzy C-means with Bi-dimensional empirical Mode decomposition for segmentation of Microarray Imge. International Journal of Computer Science issues. 2012; 9(5-3): 273-279.

[19] Zhang Guang-hua, Xiong Zhong-yang, Li Kuan, Xing Chang-yuan, Xia Shu-yin. A Novel Image Segmentation Algorithm Based on Graph Cut Optimization Problem. TELKOMNIKA (Telecommunication Computing Electronics and Control). 201; 13(4): 1337-1342. 